\title{
Morte, família e a compreensão fenomenológica: revisão sistemática de literatura
}

Death, family and the phenomenological understanding: systematic review of literature La muerte, la familia y la comprensión fenomenológica: una revisión sistemática de la literatura

\author{
Caroline Garpelli Barbosa* \\ Lígia Ebner Melchiori* \\ Carmen Maria Bueno Neme $e^{* * *}$
}

\begin{abstract}
Resumo
A pesquisa fenomenológica, ao objetivar compreender como o ser humano significa as suas experiências, permite uma investigação aprofundada de temas ligados à vida humana. Visando a compreender como a experiência da morte dentro do seio familiar tem sido abordada por estudos realizados na perspectiva fenomenológica de investigação, foi realizada uma revisão sistemática de literatura com base em resumos indexados nas bases Lilacs, PEPsic, Medline e PsycInfo, mediante o cruzamento das palavras-chave "família" (family), "morte" (death), "fenomenologia" (phenomenology). No total, foram encontrados 54 trabalhos. Em um primeiro momento, todos passaram por uma análise quantitativa e, a seguir, foram selecionados 15 para a análise qualitativa. Os resultados apontaram que os estudos realizados com embasamento na fenomenologia abordaram três grandes temas, a saber, as experiências, os significados e o enfrentamento do luto e das perdas; a relação da família com o paciente terminal; e as vivências das famílias diante de mortes repentinas.
\end{abstract}

Palavras-chave: Revisão, morte, família, fenomenologia.

\begin{abstract}
The phenomenological research aims to understand how human beings mean their experiences and allows a detailed investigation of
\end{abstract}

\footnotetext{
Texto recebido em março de 2010 e aprovado para publicação em fevereiro de 2011.

Mestranda do Programa de Pós-graduação em Psicologia do Desenvolvimento e Aprendizagem da Universidade Estadual Paulista (Unesp - campus Bauru), psicóloga, bolsista FAPESP. E-mail: psica_ca@yahoo.com.br.

** Professora doutora do Programa de Pós-fraduação em Psicologia do Desenvolvimento e Aprendizagem da Universidade Estadual Paulista (Unesp - campus Bauru).

*** Professora livre-docente do Programa de Pós-fraduação em Psicologia do Desenvolvimento e Aprendizagem da Universidade Estadual Paulista (Unesp - campus Bauru).
} 
issues related to human life. In order to understand how the death experience within the family has been addressed by studies within phenomenological research perspective, a systematic review of literature was performed based on abstracts indexed in Lilacs, PEPsic, Medline e PsycInfo, by means of the intersection of the keywords: family, death, and phenomenology. Overall, 54 papers were found. Initially, all the papers were subjected to a quantitative analysis, after which 15 were selected for qualitative analysis. The results indicated that studies with grounding in phenomenology addressed three major themes: the experiences, meanings and coping with grief and loss, the family's relationship with the terminally ill patient, and the experiences of families facing sudden death.

Keywords: Review, death, family, phenomenology.

\section{Resumen}

La investigación fenomenológica, con el objetivo de entender cómo el ser humano significa sus experiencias, permite una investigación detallada de los temas relacionados con la vida humana. Con el fin de comprender cómo la experiencia de la muerte dentro de la familia ha sido abordado por los estudios que trabajan con la metodología fenomenológica se efectuó una revisión sistemática de la literatura indizadas en LILACS, PEPsic, MEDLINE y PsycInfo, a través de la intersección de las palabras clave: familia (family), muerte (death), fenomenología (phenomenology). Se encontraron 54 estudios analizados cuantitativamente, y seleccionó 15 para el análisis cualitativo. Los resultados indicaron que los estudios con fundamento en la fenomenología abordaron tres temas principales: las experiencias, significados y manejar el dolor y da pérdida; la relación de la familia con ele enformo terminal; y las experiencias de las familias que enfrentan la muerte súbita.

Palabras clave: Revisión, muerte, familia, fenomenologia.

ideia de morte encontra-se presente na existência humana e, com ela, um conjunto de concepções relacionado às experiências, histórias de vida e vivências de cada indivíduo numa dada cultura. Sendo assim, os significados atribuídos individualmente à morte estão distantes de serem unívocos, uma vez que se relacionam, entre outros acontecimentos, à idade, às experiências familiares diante das perdas, à crença religiosa ou fé, à cultura, bem como a convicções e valores pessoais e sociais (Kovács, 2002). 
Pesquisas realizadas tanto com crianças como com adolescentes que passaram por perdas em decorrência da morte de pessoas significativas em suas famílias têm mostrado que a ausência de suporte nos momentos de luto pode gerar repressão de sentimentos de tristeza, conflito consigo mesmo e com os demais membros da família, dificuldade de atenção nas aulas, problemas de comportamento e distúrbios psicossomáticos (Mufson, 1985; Schwab, 1997; Domingos \& Maluf, 2003; Horsley \& Patterson, 2006; Packman, Horsley, Davies \& Kramer, 2006; Jucá et al., 2007). Tais sintomas podem surgir por não saberem como trabalhar com sua própria dor e não terem um suporte adequado para lidar com seus medos e angústias. Nesse sentido, observam-se sérias consequências decorrentes de um luto mal elaborado, no qual a experiência da perda se dissocia dos sentimentos e emoçôes, trazendo efeitos que podem ser agravados nos casos em que a morte ocorre dentro do sistema familiar.

Dada a relevância que a perda de um membro da família tem tanto para o indivíduo como para todo o sistema familiar, bem como os efeitos e implicações das diferentes formas de se lidar e de se compreender a morte, considera-se a necessidade de estudos que propiciem a abordagem e a reflexão sobre como ela é vivenciada no âmbito individual e familiar, uma vez que a experiência da perda por morte será inevitável em algum momento da trajetória de vida de todo ser humano. Segundo KüblerRoss (1991), somente a partir do momento em que as pessoas tiverem espaço para refletir sobre a própria morte e o morrer é que poderão encará-la de maneira mais saudável e quanto antes um indivíduo puder falar sobre a morte, menor será seu espanto diante dela. Para tanto, considera-se relevante saber qual o panorama atual dos estudos sobre a morte, especialmente os que buscam compreender concepçóes e vivências de pessoas que, por seus relatos ou depoimentos, mostram como se tem lidado com esse tema no mundo contemporâneo.

Nas últimas décadas, o tema da morte vem sendo alvo de diversas pesquisas em diferentes áreas do conhecimento, o que mostra a complexidade do tema e sua natureza interdisciplinar. Entre esses estudos, as pesquisas qualitativas se mostram fontes ricas de conhecimento acerca da experiência humana, pois se apresentam como uma possibilidade mais ampla de compreender os significados e sentidos que o homem produz em sua coexistência com o mundo e com as pessoas ao redor. Entre esses modelos de pesquisa, a fenomenologia se apresenta como um método capaz de possibilitar a compreensão dos significados que o ser humano confere às suas experiências, permitindo, assim, a realização 
de um aprofundamento nas vivências dos colaboradores, especialmente quando se trata de temas estreitamente ligados às questôes da existência (Forghieri, 1997; Valle, 1997). Com isso, nos estudos referentes à relação do homem com a morte, esse modelo de pesquisa permitirá que se vá além de considerações teóricas ou de discussões de aspectos periféricos que costumam cercar as reflexóes sobre esse difícil tema, para que se possa compreendê-lo no interior da vida humana.

Assim, considerando-se a profundidade que o método fenomenológico proporciona no estudo das vivências, bem como se levando em conta que é no contexto familiar que se constitui grande parte da subjetividade humana, esta pesquisa tem como objetivo compreender, com base numa revisão sistemática da literatura, como a experiência da morte dentro do seio familiar tem sido abordada em estudos realizados na perspectiva fenomenológica de investigação.

\section{Método}

Procedimento para a coleta de dados

Com o intuito de cumprir com o objetivo proposto, foi realizada uma busca sistemática de resumos de artigos indexados nas bases de dados Lilacs (Literatura Latino Americana de Ciências de Saúde), PEPsic (Periódicos Eletrônicos em Psicologia), Medline (Medical Literature Analysis and Retrieval System Online) e PsycInfo, mediante o cruzamento das palavras-chave "família" (family) e "morte" (death) e "fenomenologia" (phenomenology). Os critérios de inclusão utilizados foram: a) veículo de publicação: periódicos indexados, livros e resumos de dissertação; b) período: 1997 a 2009 - critério adotado a fim de seguir o padrão estabelecido pelas bases de dados Lilacs e Medline, em que o tempo mínimo de busca abrange esse período; c) idioma de publicação: português, inglês ou espanhol; d) modalidade de produção científica: estudos empíricos, teóricos e de revisão bibliográfica.

\section{Procedimento para a análise dos dados}

A análise dos dados foi realizada em duas etapas: a primeira, quantitativa; e a segunda, qualitativa. Para a análise quantitativa, todos os resumos encontrados foram agrupados conforme: a) o número de artigos publicados por ano (nacionais e internacionais); b) a natureza da pesquisa (relato de pesquisa, revisão, teórica); c) do veículo de publicação 
(periódicos, livros, outros); d) do país de publicação. Posteriormente a esses passos, foram excluídos os resumos repetidos e os que não explicitavam a natureza da pesquisa.

$\mathrm{Na}$ análise qualitativa, foram considerados apenas os resumos que abordavam a temática da família e da morte por meio do método fenomenológico de investigação. Com esses trabalhos, foi realizada uma análise com base nos passos sugeridos por Martins e Bicudo (1989), que envolvem: a) leitura atenta, a fim de se obter um panorama geral dos trabalhos; b) apreensão dos temas abordados; c) busca de convergências e divergências entre as temáticas de estudo; d) apreensão de categorias temáticas, de modo a agrupar trabalhos semelhantes; e) apresentação e descrição das várias categorias temáticas apreendidas, a fim de evidenciar tendências e lacunas nos estudos encontrados.

\section{Resultados}

\section{Análise quantitativa}

Foram encontrados 54 resumos (35 na PsycInfo, 14 na Medline, cinco na Lilacs e nenhum na PEPsic) publicados no período de 1997 a 2009. Destes, seis foram excluídos: um por não deixar claras a natureza da pesquisa e a metodologia utilizada, e cinco por serem repetidos, restando, portanto, 48 trabalhos. Dos 48 resumos analisados, as publicações internacionais englobaram $90 \%$ dos estudos $(n=43)$, apresentando 38 relatos de pesquisa, dois estudos teóricos e três de revisão de literatura. Foram 30 publicações em periódicos $(21 \mathrm{em}$ revistas ou jornais dos Estados Unidos, cinco em periódicos do Reino Unido, dois da Austrália, um da Coreia do Sul e um da Turquia), 12 resumos de dissertaçóes (todos publicados nos Estados Unidos) e um em livro (Estados Unidos). Com relação às publicações nacionais, elas corresponderam a 10\% (n = 5) do total de trabalhos publicados, sendo que, destes, todos eram relatos de pesquisa publicados em periódicos veiculados no Brasil e América Latina.

Nas bases nacionais, observou-se ausência, até o ano 2006, de publicações de trabalhos relacionando os temas morte, família e fenomenologia, o que pode sugerir que o interesse por essa temática tenha surgido mais recentemente na literatura latino-americana, diferentemente do que ocorreu com as publicações internacionais, que apresentaram estudos desde 1997 ( $\mathrm{n}=4$ ), com maior incidência de trabalhos em $2006(\mathrm{n}=8)$. 
A tabela 1 descreve as publicações encontradas de acordo com o ano de publicação, nacionalidade e natureza da pesquisa.

\section{Tabela 1}

Publicações nacionais e internacionais no período de 1997 a 2009 segundo ano de publicação e natureza da pesquisa

\begin{tabular}{c|c|c|c|c|c|c|c}
\hline \multirow{2}{*}{$\begin{array}{c}\text { Ano de } \\
\text { publicação }\end{array}$} & \multicolumn{3}{|c|}{ Nacional } & \multicolumn{3}{|c|}{ Internacional } & \multirow{2}{*}{ Total } \\
\cline { 2 - 7 } & $\begin{array}{c}\text { Relato } \\
\text { Pesq. }\end{array}$ & Teórico & Revisão & $\begin{array}{c}\text { Relato } \\
\text { Pesq. }\end{array}$ & Teórico & Revisão & \\
\hline 2009 & 2 & - & - & 4 & - & 1 & 7 \\
2008 & - & - & - & 6 & 1 & 1 & 8 \\
2007 & 1 & - & - & 5 & - & - & 6 \\
2006 & 2 & - & - & 3 & - & - & 5 \\
2005 & - & - & - & 2 & 1 & - & 3 \\
2004 & - & - & - & 1 & - & 1 & 2 \\
2003 & - & - & - & 1 & - & - & 1 \\
2002 & - & - & - & 3 & - & - & 3 \\
2001 & - & - & - & 3 & - & - & 3 \\
2000 & - & - & - & 3 & - & - & 3 \\
1999 & - & - & - & 2 & - & - & 2 \\
1998 & - & - & - & 1 & - & - & 1 \\
1997 & - & - & - & 4 & - & - & 4 \\
\hline Total & 5 & 0 & 0 & 38 & 2 & 3 & 48 \\
\hline
\end{tabular}

Análise qualitativa

Com base na leitura dos 48 trabalhos remanescentes, observou-se que, embora tenham sido encontrados por meio das palavras-chave "morte", "família" e "fenomenologia", sete não utilizaram a fenomenologia como método, apesar de terem como foco de pesquisa a "morte" e a "família"; 17 empregaram o método fenomenológico, mas o objeto de estudo da pesquisa não relacionou "morte e família", e sim "morte" ou "família", ou ainda outra população; e nove trabalhos, além de não utilizarem a metodologia fenomenológica, também não abordavam a morte e a família como foco do estudo. Restaram, portanto, 15 resumos que utilizaram o método fenomenológico para investigar a temática "morte e família" como objetivo principal do estudo, correspondendo a $31 \%$ dos trabalhos encontrados.

Mediante exame dos trabalhos submetidos à análise qualitativa, foi possível observar que os trabalhos realizados com embasamento na 
fenomenologia abordaram três grandes subáreas, agrupadas nas categorias temáticas a seguir: a) Experiências, significados e enfrentamento do luto e das perdas; b) A família e o paciente terminal; e c) Mortes inesperadas e seus impactos.

\section{a) Experiências, significados e enfrentamento do luto e das perdas}

Foram encontrados oito trabalhos com o objetivo de verificar as experiências, significados e enfrentamento do luto e das perdas após a morte de um dos membros da família. Os artigos consultados abordaram o assunto pelo ponto de vista de adultos/idosos (sete estudos) e crianças (um estudo).

Entre os trabalhos realizados com adultos, obtiveram-se três relacionados à experiência de perda do cônjuge. O trabalho de Doherty e ScannellDesch (2008) buscou descrever a experiência de mulheres que ficaram viúvas durante a gravidez, ao terem seus maridos mortos no ataque terrorista de 11 de setembro ou enquanto serviam no exército dos Estados Unidos nas guerras do Afeganistão e Iraque. As vivências identificadas durante o processo da gravidez e do luto foram agrupadas nas seguintes categorias: a) Negação versus barganha com a realidade: ele não vindo para casa; b) Atravessando a gravidez: voo solitário no vazio; c) Planejando o nascimento: reunindo meu time; d) Minha rede de segurança: um grupo de irmãs; e) Jornada não planejada: uma amarga e doce volta para casa; f) Pessoas próximas: rede de família e amigos; g) Ausência de pessoas: ser abandonado pelos outros; e h) Recriando o lar: um novo normal. Os relatos das participantes descreveram as emoções, vulnerabilidades e os desafios experimentados ao depararem a ausência dos maridos no momento da gravidez. Tal estudo proporciona profunda compreensão sobre as vivências dessas mulheres e contribui para futuras intervenções com pessoas em situações similares.

O trabalho de Boswell (2008) foi realizado com homens e mulheres heterossexuais e homossexuais, e visou a identificar, descrever e comparar as estruturas do sofrimento resultante do luto pela morte do cônjuge nas populações referidas. A fim de atingir esse objetivo, além do método fenomenológico, também foram utilizados os instrumentos Inventory of Complicated Grief; Hogan Grief Reaction Checklist; Perceived Social Support Questionnaire for Family, Friends and In-laws; e o NEO FiveFactor Inventory. Os resultados desse estudo indicaram que a maior parte dos viúvos e viúvas homossexuais recebeu menos apoio social e foram 
mais privados de expressar seu sofrimento, revelando que o cônjuge homossexual sofre com o preconceito e parece não ter os mesmos direitos de chorar o luto do cônjuge como nos casais heterossexuais, entre os quais o sofrimento foi amenizado com o suporte social.

Evans (2001) realizou um estudo com sete idosas viúvas, com idades entre 70 e 88 anos, e que tinham sido casadas, no mínimo, durante 30 anos. Procurou abordar questôes referentes à reformulação da identidade, transformação pessoal, e ao significado da vida após a perda de seus cônjuges. Os resultados revelaram que nenhuma das colaboradoras da pesquisa referiu a si mesma como viúva. Elas veem a si mesmas com base nos papéis sociais que desempenham na sociedade e na família, das características e atributos pessoais que têm, das dúvidas que têm quanto ao futuro e ao avanço da idade, da filosofia de vida, da saúde e também dos relacionamentos mantidos com outras pessoas, especialmente com a família. Com relação às transformações, elas estiveram relacionadas às perdas, não só a do cônjuge, mas também as de outras pessoas queridas, de habilidades físicas, energia e saúde. O significado da vida após a morte do cônjuge esteve relacionado às relações e conexões estabelecidas com o círculo social (família, amigos, trabalhos voluntários) e também com o falecido por meio de lembranças, fotografias e rituais espirituais.

Entre os estudos com adultos, o trabalho de Bielek (2008) procurou compreender as vivências de casais de genitores antes, durante e após a morte de um filho, de modo a abordar, especialmente, como é a última memória que eles têm da criança. Os resultados apontaram que, para esses genitores, o tempo vivido com a criança foi insuficiente para dizerem ao filho tudo o que sentiam. Por outro lado, a percepção da insuficiência do tempo, levouos a tentarem aproveitar ao máximo todos os momentos ao lado do filho para expressarem seu amor por ele e estarem juntos. Como última memória, os genitores recordaram apenas dos bons momentos vividos junto ao filho, principalmente quando ele ainda estava saudável.

Quanto aos trabalhos com adultos, apenas um abordou a morte de genitores. O estudo de Daugherty (2002) buscou compreender como os adultos veem suas experiências de terem perdido um dos genitores durante a infância ou a adolescência. Para isso, 40 pessoas foram entrevistadas, desvelando oito categorias de significados: "Eu vejo minha perda como uma fabricante de duas vidas"; "Eu vivo com memórias de minha perda na infância"; "Eu vejo a minha perda através de olhos adultos"; "Eu tenho uma constante consciência da mortalidade"; "Eu sinto a ausência de meu pai/mãe ao longo da minha vida"; 
"Meu pai/mãe permanece presente em minhas memórias"; "Compreensão e definição de quem eu sou"; "Eu entendo a influência da minha perda em minha família". Os resultados apontaram para o forte impacto que exerce a morte dos pais na trajetória de vida individual e familiar daqueles que vivenciam essa perda.

A pesquisa de Houwarth (2008) visou a compreender as vivências de seis adultos que nasceram após a morte de um irmão. Como resultados, quatro categorias temáticas foram encontradas: "Aprendizagem sobre o irmão"; "Experienciando o irmão"; "Vivendo em um sistema familiar impactado"; e "Integrando a experiência". O estudo sugere que a morte de uma criança pode atingir o filho subsequente de maneira significativa. Pessoas com essas experiências podem apresentar um sentido pessoal de perda, bem como se sentirem impactadas de maneira secundária pelos múltiplos efeitos gerados dentro do sistema familiar. De maneira geral, indivíduos que nasceram após a morte de um irmão podem acreditar que tal morte influenciou direta ou indiretamente o desenvolvimento de suas vidas emocionais e também o modo como se relacionam com os outros.

Com o objetivo de explorar a experiência de indivíduos enlutados e os efeitos que essas perdas tiveram em sua saúde mental e física, Douglas (2004) entrevistou 12 pessoas que haviam perdido noivo(a), esposo(a) ou filho(a) há pelos menos três meses antes da realização do estudo. Como resultado, as seguintes categorias foram encontradas: a) "Eu me sinto devastado e sozinho"; b) "Isso me machuca o tempo todo, essa dor está sempre comigo"; c) "Eu me sinto vazio e com raiva"; d) "Eu não quero continuar vivendo". Os dados mostram que essas pessoas, muitas vezes, relatam experimentar profundas dores emocionais e físicas que as afetam em suas vidas cotidianas, o que, em alguns casos, gera depressão e ideações suicidas.

O estudo de Andrews e Marotta (2005) objetivou explorar como a espiritualidade (recurso utilizado pelos adultos para o enfrentamento) pode auxiliar crianças em sofrimento pela perda de algum membro da família. Como resultado, concluíram que a maior diferença entre reaçôes de crianças e adultos parece ser quanto ao como e ao quando as crianças fazem as significações das perdas. A maior similaridade entre adultos e crianças foi o papel essencial que as relações interpessoais têm na facilitação da superação dos sofrimentos.

\section{b) A família e o paciente terminal}

Entre os trabalhos que abordaram a temática da família diante do 
adoecimento e da terminalidade, um foi realizado com idosos e seus familiares, um abordou a família diante do diagnóstico de morte cerebral e dois focaram a convivência com o paciente terminal.

O trabalho de Leichtentritt e Rettig (2002) pretendeu revelar e interpretar as crenças de idosos israelenses e seus familiares, concernentes às decisões que envolveriam prolongamento da vida ou antecipação da morte diante do sofrimento. A premissa do estudo é de que seria possível identificar crenças familiares involuntárias, que foram construídas coletivamente por seus membros e que provavelmente interfeririam no momento dessas decisões. Os resultados corroboraram essa premissa.

O trabalho desenvolvido por Sales, Molina e Cardoso (2006) buscou apreender os sentimentos da família que presta cuidados a um ente amado com câncer. A partir dos relatos analisados, duas grandes categorias foram obtidas: a) "vivenciando a facticidade de conviver com o câncer em seu domicílio"; e b) "vivenciando a morte de um ente querido". A concepção dos familiares revelou que o cuidar de alguém querido e com uma doença considerada fatal lança os familiares em um mundo de sentimentos paradoxais decorrentes da necessidade de ter de prestar cuidados, ao mesmo tempo em que necessitam abarcar em si sua dor.

Mcdonald (1998), em seu trabalho com pacientes terminais, familiares e funcionários de hospitais que cuidam desses pacientes, buscou compreender o sentido de comunidade desenvolvido durante os processos de morte, especificamente dentro dos hospitais. Dentro desse contexto, os resultados sugeriram que fortes relações interpessoais e sistemas de apoio baseados em relações de intimidade entre pacientes, família e equipe de saúde tornam a transição para a morte um evento mais significativo e também doloroso. $\mathrm{O}$ estudo também mostrou que cada morte é uma experiência única para todos aqueles que estão próximos da pessoa que está morrendo e que o fato de estarem dentro de um hospital não determinou a presença ou ausência do senso de comunidade entre as três famílias estudadas.

Frid; Haljamäe; Öhlén e Bergbom (2007) estudaram 17 familiares de pacientes que foram diagnosticados com morte cerebral, a fim de descrever a experiência desses participantes frente ao recebimento dessa notícia. Seis categorias descritivas foram obtidas para mostrar a imagem das experiências de ser confrontado pelo diagnóstico de morte cerebral de um familiar próximo: a) "irrealidade caótica"; b) "colapso interno"; c) "sentimento de abandono"; d) "agarrando-se à esperança de sobrevivência"; e) "reconciliação com a realidade da morte”; f) "recebendo cuidado que dá conforto". Esses resultados revelaram 
que a experiência de ser informado sobre a morte cerebral de alguém remete a sentimentos confusos, pois, em muitos casos, há a sensação de que a pessoa continua viva fisicamente, apesar do diagnóstico de irreversibilidade da morte. Daí a importância de que os profissionais de saúde estejam preparados para esclarecerem as dúvidas da família, bem como oferecerem cuidados a eles nesse momento vivido com ambivalência.

\section{c) Mortes inesperadas e seus impactos}

As mortes inesperadas foram abordadas em três trabalhos, dois realizados com genitores que perderam seus filhos de maneira repentina e um que estudou o suicídio e seu impacto na família.

O estudo desenvolvido por Gudmundsdottir e Chesla (2006) abordou sete famílias (15 indivíduos), com o intuito de descrever e compreender hábitos e práticas desenvolvidas na significação da morte repentina e inesperada de seus filhos. Para isso, os participantes foram entrevistados juntos e individualmente. Os resultados mostraram que a cicatrização da dor da família apenas foi possível quando os genitores se envolveram em práticas que os auxiliaram a reconhecer a morte dos filhos, bem como lhes permitiram estabelecer uma conexão contínua com eles, de modo a integrá-los e mantê-los vivos em suas vidas de alguma maneira.

Ainda com relação à morte repentina de filhos, Gudmundsdottir (2009) investigou como a dor emocional em decorrência da perda também se reflete em dores corporais para os genitores dessas crianças. Os dados apontaram para diversas manifestações psicossomáticas durante a fase do luto, ressaltando a importância de também testemunhar e reconhecer as histórias corporais no trabalho clínico com essa população que ainda está aprendendo a viver em um mundo sem o filho amado.

Fielden (2003), com o objetivo de explorar e interpretar as experiências vividas por seis pessoas que perderam um membro familiar próximo por suicídio, apontou que as categorias reveladas mostraram que sobreviver ao suicídio de alguém próximo foi um processo transformador para a descoberta de novas formas de compreensão e de relação com o mundo.

\section{Discussão}

No período examinado (1997 a 2009), foram encontrados apenas 15 estudos sobre morte e família que utilizaram a metodologia fenomenológica na pesquisa. Considerou-se que se trata de uma frequência relativamente 
baixa de publicações, visto que corresponde a uma média de pouco mais de um trabalho publicado por ano. Entretanto, foi possível observar que houve aumento no número de publicaçôes sobre a morte nos últimos anos, indicando que, a despeito das dificuldades ainda existentes em se abordar o assunto, o tema vem sendo cada vez mais estudado em diferentes países. No Brasil, contudo, apenas cinco trabalhos foram publicados sobre a temática pesquisada, sendo que, destes, dois abordaram de maneira secundária a questão da morte na família, tocando a questão somente como fruto de discussões e resultados de estudos sobre o câncer e a hospitalização.

A análise qualitativa, por sua vez, revelou que os trabalhos desenvolvidos com base na metodologia fenomenológica abordaram a morte, ora junto a famílias enlutadas ou que já tiveram alguma perda significativa, e ora junto àquelas que experimentaram a condição de terminalidade de um de seus membros. Além disso, observou-se predomínio de estudos realizados com adultos, o que pode ser um reflexo da ideia propagada na sociedade de que crianças e adolescentes não se preocupam ou não são capazes de compreender o assunto (Vendruscolo, 2005; Borges et al., 2006), ou ainda, da dificuldade que os adultos relatam sentir quando precisam conversar com seus filhos sobre a morte (Domingos \& Maluf, 2003; Jucá et al., 2007).

Os dados apontam que as pesquisas fenomenológicas que cercam o tema têm enfatizado, sobretudo, a compreensão dos significados atribuídos ao impacto que a morte gera na família que se encontra na iminência de perder ou da que já perdeu um de seus entes queridos. Diante disso, ao tentar reconstruir as experiências das pessoas entrevistadas, observa-se que a metodologia fenomenológica se apresentou não apenas como uma ferramenta de coleta de dados, mas também criou espaço para que os colaboradores entrevistados expressassem suas reflexões e sentimentos sobre as perdas que tiveram, bem como compartilhassem a sua dor. No entanto, cabe ressaltar que os estudos encontrados, apesar de promoverem uma ampliação na compreensão de como as pessoas significam suas experiências diante das perdas, não abordaram como a perspectiva da morte, como fenômeno inerente à vida de cada um dos entrevistados, é vivenciada no cotidiano, ou seja, para além da experiência do adoecimento, do luto e de seus impactos. A condução de pesquisas nessa direção, tal como a realizada por Barbosa (2010), propicia a condução de diálogos pouco comuns, conforme abre espaço para que um assunto tão pouco abordado seja não apenas relatado e refletido, mas também vivido e discutido no dia a dia das famílias. No estudo realizado pela autora, as entrevistas fenomenológicas realizadas com pessoas de diferentes idades e pertencentes a uma mesma família e que não tivessem experimentando o 
luto, além de terem exercido função terapêutica para diversos colaboradores, também lhes abriu novas possibilidades de olhar para a própria existência e de refletir sobre a relação que mantêm com a morte e com as perdas.

\section{Considerações finais}

Os estudos fenomenológicos analisados, conforme buscam penetrar o mundo de cada pessoa e cada família em particular, visando a se aproximar de quais são suas principais inquietações no que concerne ao relacionamento com as perdas, trazem subsídios importantes para que se possa compreender, clarificar e refletir sobre como a sociedade atual se relaciona com a morte. Nesse sentido, a partir das lacunas encontradas nessa literatura, sugere-se que novos trabalhos realizados nessa abordagem busquem aprofundar como a morte como fenômeno inerente à condição humana tem sido concebida e significada no contexto familiar contemporâneo, uma vez que tal conhecimento pode favorecer discussões e reflexões que permitam devolver a morte ao seu lugar mais originário, a saber, o interior da vida humana.

\section{Referências}

Andrews, C. R. \& Marotta, S. A. (2005). Spirituality and coping among grieving children: a preliminary study. Counseling and Values, 50 (1), 38-50.

Barbosa, C. G. (2010). A família e a morte: estudo fenomenológico com adolescentes, genitores e avós. Dissertação de Mestrado, Programa de PósGraduação em Psicologia do Desenvolvimento e Aprendizagem, Universidade Estadual Paulista, Bauru.

Bielek, S. (2008). Goodbye seems to be the hardest word: a phenomenological analysis of parents' anticipatory mourning of a child's impending death. Dissertation Abstracts International: Section B: The Sciences and Engineering, 69 (5-B), 3259.

Borges, A. D. V. S. et al. (2006). Percepção da morte pelo paciente oncológico ao longo do desenvolvimento. Psicologia em Estudo, Maringá, 11 (2), 361-369.

Boswell, C. (2008). A phenomenological study of the experience of grief resulting from spousal bereavement in heterosexual and homosexual men and women. Dissertation Abstracts International: Section B: The Sciences and Engineering, 68 (7-B), 4811. 
Daugherty, D. J. (2002). When death affects life: the experience of adults who live with the loss of a parent that died in childhood or adolescence. Dissertation Abstracts International Section A: Humanities and Social Sciences, 62 (12-A), 4347.

Doherty, M. E. \& Scannell-Desch, E. (2008). The lived experience of widowhood during pregnancy. Journal of Midwifery Women's Health, 53 (2), 103-109.

Domingos, B. \& Maluf, M. R. (2003). Experiências de perda e de luto em escolares de 13 a 18 anos. Psicologia: Reflexão e Crítica, 3 (16), 577-589.

Douglas, D. H. (2004). The lived experience of loss: a phenomenological study. Journal of the American Psychiatric Nurses Association, 10 (1), 24-32.

Evans, K. A. (2001). Resilient older widows: a phenomenological study of identity reformulation, personal transformation, and re-creation of life meaning following loss of a longtime spouse. Dissertation Abstracts International: Section B: The Sciences and Engineering, 62 (5-B), 2515.

Fielden, J. M. (2003). Grief as a transformative experience: weaving through different lifeworlds after a loved one has completed suicide. International Journal of Mental Health Nursing, 12 (1), 74-85.

Forghieri, Y. C. (1997). Psicologia fenomenológica: fundamentos, método e pesquisa. São Paulo: Pioneira.

Frid, I. et al. (2007). Brain death: close relatives' use of imagery as a descriptor of experience. Journal of Advanced Nursing, 58 (1), 63-71.

Gudmundsdottir, M. \& Chesla, C. A. (2006). Building a new world: habits and practices of healing following the death of a child. Journal of Family Nursing, 12 (2), 143-164.

Gudmundsdottir, M. (2009). Embodied grief: bereaved parents' narratives of their suffering body. Omega: Journal of death and dying, 59 (3), 253-269.

Horsley, H. \& Patterson, T. (2006). The effects of a parent guidance intervention on communication among adolescents who have experienced the sudden death of a sibling. The American Journal of Family Therapy, 34, 119-137.

Houwarth, H. H. (2008). Ghost in the family: an interpretative phenomenological analysis of the experiences of individuals born after the death of a sibling. Dissertation Abstracts International: Section B: The Sciences and Engineering, 69 (3-B), 1956. 
Jucá, V. J. S. et al. (2007). Significando a morte, através de redes sociais, em um contexto de vulnerabilidade social - um estudo com crianças préescolares, seus pais e professores. Psicologia e Sociedade, (19), 122-130.

Kovács, M. J. (2002). Morte e desenvolvimento humano. São Paulo: Casa do Psicólogo.

Kübler-Ross, E. (1991). Sobre a morte e o morrer: o que os doentes terminais têm para ensinar a médicos, enfermeiros, religiosos e aos seus próprios parentes. São Paulo: Martins Fontes.

Leichtentritt, R. D. \& Rettig, K. D. (2002). Family beliefs about end-of-life decisions: an interpersonal perspective. Death Studies, 26 (7), 567-594.

Martins, J. \& Bicudo, M. A. V. (1989). A pesquisa qualitativa em psicologia: fundamentos e recursos básicos. São Paulo: EDUC.

Mcdonald, J. E. (1998). The spirit of meaning through the surrender and catch of death: a phenomenological study of three hospice families. Dissertation Abstracts International: Section B: The Sciences and Engineering, 59 (5-B), 2108.

Mufson, T. M. S. W. (1985). Issues surrounding sibling death during adolescence. Child and adolescenct social work journal, 4 (2), 204-218.

Packman, W; Horsley, H.; Davies, B. \& Kramer, R. (2006). Sibling bereavement and continuing bounds. Death Studies, 30, 817-841.

Sales, C. A.; Molina, M. A. S. \& Cardoso, R. C. S. (2006). Estar com um ente querido com câncer: concepçôes dos familiares. Nursing, 97 (8), 878882.

Schwab, R. (1997). Parental mourning and chlidren's behavior. Journal of counseling and development, 75, 258-265.

Valle, E. R. M. (1997). Um estudo das pesquisas psicológicas na abordagem fenomenológica sobre o câncer infantil. In: E. R. M. Valle (org.). Câncer infantil: compreender e agir. (pp. 73-112). Campinas: Psy.

Vendruscolo, J. (2005). Visão da criança sobre a morte. Revista Medicina, 38 (1), 26-33. 